\title{
Editorial
}

Onkologe $2021 \cdot 27: 522-523$

https://doi.org/10.1007/s00761-021-00954-3

Angenommen: 9. April 2021

๑) Springer Medizin Verlag GmbH, ein Teil von Springer Nature 2021

\author{
K. Höffken ${ }^{1} \cdot$ C. Bruns ${ }^{2} \cdot$ H. Schmidberger ${ }^{3}$ V. Stratmann ${ }^{4}$ \\ ${ }^{1}$ Berlin, Deutschland \\ ${ }^{2}$ Klinik und Poliklinik für Allgemein-, Viszeral-, Tumor- und Transplantationschirurgie, Unikilinik Köln, Köln, \\ Deutschland \\ ${ }^{3}$ Klinik und Poliklinik für Radioonkologie, Universitätsmedizin Mainz, Mainz, Deutschland \\ ${ }^{4}$ Springer-Medizin Verlag $\mathrm{GmbH}$, Heidelberg, Deutschland
}

\section{Was gibt es Neues?}

Liebe Leserinnen, liebe Leser,

Sie werden sich vielleicht fragen, warum Herausgeber und Verlag beschlossen haben, eine neue Rubrik „Update Onkologie" für unsere Fachzeitschrift zu etablieren.

Sind die Beiträge der Leitthemen etwa kein Update des jeweiligen Themas? Selbstverständlich sind sie das! Aber die Leitthemenhefte gehen in ihren Beiträgen auf die neuesten Kenntnisse des Leitthemas aus der Sicht der verschiedenen onkologischen Fachdisziplinen ein - also interdisziplinär.

Stattdessen sollen in der Rubrik „Update Onkologie" die Fortschritte des jeweiligen Fachgebiets beschrieben werden. Dabei kommen die Mitglieder des Beirats unserer Fachzeitschrift in jährlichen Abständen zu Wort, um die Fortschritte ihres onkologischen Fachgebiets zu beschreiben und damit den Leserinnen und Lesern die Dynamik in Forschung und Krankenversorgung zur Kenntnis zu geben.

Wir beginnen in diesem Heft mit dem Fachgebiet der Ophthalmoonkologie.

Aus der Arbeitsgruppe von Antonia Joussen von der Charité berichten Aline Isabel Riechardt und Mitautoren am Beispiel des Uveamelanoms über die Fortschritte in Diagnostik und Therapie und damit schwerpunktmäßig über die Dynamik der Therapieforschung von Tumorerkrankungen des Auges.

Es folgen in den nächsten Heften die Fortschrittsberichte der anderen, den Beirat bildenden, Fachvertreter.
Wir sind überzeugt, dass durch diese Übersichten ein guter Einblick in die onkologischen Perspektiven der an der Versorgung von Krebspatienten beteiligten Disziplinen gewonnen werden kann.

K. Höffken, C. Bruns, H. Schmidberger Herausgeber

\section{Stratmann \\ Verlagsredaktion}

\section{Korrespondenzadresse}

Prof. Dr. K. Höffken

Welserstraße 21, 10777 Berlin, Deutschland hoeffken.editor@gmail.com

Interessenkonflikt. K. Höffken, C. Bruns, H. Schmidberger und V. Stratmann geben an, dass kein Interessenkonflikt besteht. 
Hier steht eine Anzeige.

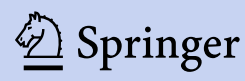

